\title{
Polarization compensation of Fresnel aberrations in telescopes
}

\author{
Natalie Clark ${ }^{\mathrm{a}}$ and James B. Breckinridge ${ }^{\mathrm{b}}$ \\ ${ }^{\mathrm{a}}$ NASA Langley Research Center, Hampton Virginia \\ ${ }^{\mathrm{b}}$ CALTECH \& College of Optical Science, University of Arizona
}

\begin{abstract}
Large aperture space telescopes are built with low F\#'s to accommodate the mechanical constraints of launch vehicles and to reduce resonance frequencies of the on-orbit system. Inherent with these low F\# is Fresnel polarization which effects image quality. We present the design and modeling of a nano-structure consisting of birefringent layers. Analysis shows a device that functions across a 400nm bandwidth tunable from 300nm to $1200 \mathrm{~nm}$. This Fresnel compensator device has a cross leakage of less than 0.001 retardance.
\end{abstract}

Keywords: Polarization, exoplanet, telescope, birefringence, polyimide,wavefront, adaptive optics, and phase.

\section{INTRODUCTION}

Direct imaging of terrestrial exoplanets requires a telescope/instrument system capable of controlling and suppressing unwanted star light to approximately one part in $10^{12}$ over a wide wavelength bandpass spanning the UV to near IR spectrum $^{1-4}$. Terrestrial exoplanets are faint, hence require large aperture telescopes to record their image. Because of the required size of the aperture ( 3 to 8 meters), coronagraph designs that fit into launch vehicles employ low F\# (typically 1 to 1.5) to collect and focus the radiation. These low F\# systems require a steeply curved mirror that introduce spatially varying polarization effects which in turn reduces system transmittance and increases scattered light. The polarization effects associated with metals and anisotropies have been largely overlooked in exoplanet missions. This paper characterizes the polarization affects and presents our Spatially Variable Retardance Plate (SVRP) as a method to compensate for the Fresnel polarization effects.

The properties of metal and dielectric anisotropic coatings have been largely overlooked in the analysis and performance characterization of low F\# telescopes for exoplanet missions ${ }^{5}$. Several coronagraph architectures have been proposed ${ }^{1-4}$. Each one of these will have degraded performance because of Fresnel polarization apodization. This paper addresses the modeling, and mitigation of Fresnel polarization effects in space-based coronagraphic telescopes using an innovative device that incorporates birefringent nanolayers ${ }^{6}$ to control the amplitude and phase of polychromatic white light wavefronts. The SVRP device provides control of the broadband white-light optical polarization and retardation to maximize coronagraph system transmittance, contrast, and to minimize losses to scattered light. As discussed in Section 2 this device can be located at a much smaller pupil plane ( $10-\mathrm{cm}$ diameter) which will be much smaller, lighter, and easier to fabricate than an optical element located on or next to the primary mirror.

An understanding of the sources of partial polarization in full-scale coronagraphs is necessary for accurate performance modeling, specification of mirrors and coatings, and to design necessary alternatives to mitigate the effects of this internal polarization produced by Fresnel effects. Scattered light control to better than one part in $10^{9}$ has been demonstrated $^{1-4}$ over a $10 \%$ optical bandwidth using wavefront sensing and control with an adaptive optics device in the High Contrast Imaging Test bed (HCIT) located in a vacuum system at JPL. However, this system functions at an Fnumber 28.5 and does not include an optic representative of a large (2-8 m), low F\# primary mirror characteristic of modern space coronagraph designs (ATLAST, PECO, EPIC, ACCESS, TPF-C).

UV/Optical/IR Space Telescopes and Instruments: Innovative Technologies and Concepts V, edited by Howard A. MacEwen, James B. Breckinridge, Proc. of SPIE Vol. 8146, 814600 - ( 2011 SPIE CCC code: $0277-786 X / 11 / \$ 18 \cdot$ doi: $10.1117 / 12.896638$ 


\section{Coronagraph System Considerations}

A schematic of an imaging coronagraph telescope system is shown in Figure 1. Referring to the figure, the chief ray is dotted and the marginal ray is dashed. The location where the marginal ray crosses the optic axis is an image plane. The location where the chief ray crosses the optic axis is the pupil plane (an image of the pupil). As with most astronomical telescopes the entrance pupil is co-located with the telescope aperture. Powered optical elements are shown at planes 1 , 3 and 5. The ring mask, shown at plane 4 is not a powered element. Although the telescope primary may be quite large, 8 meters as planned in NASA exoplanet missions ${ }^{2,8}$, the mitigation of Fresnel Polarization can be done at a much smaller and convenient pupil plane such as at plane 4 where the ring mask is located. The functions of the polarization compensator and ring mask can be combined. The diameter of the pupil in the NASA testbeds systems is on the order of $3 \mathrm{~cm}$. For the final exoplanet missions using $8 \mathrm{~m}$ telescope the ring mask would be much larger than $3 \mathrm{~cm}$. Our LaRC Optical Nanomaterials Synthesis and Fabrication Laboratory (ONSL) facilities can support fabrication of devices with 50nm position resolution, 0.6 micron feature sizes for advanced optical optic devices of even meters in diameter. Because the key fabrication equipment is digitally controlled, devices can be fabricated inexpensively and rapidly facilitating design refinements and modifications. Hence, this facility can support fabrication of the polarization compensation device throughout the exoplanet mission from demonstration in one of the NASA coronagraph systems proposed under this effort all the way to the final flight device. Direct imaging of terrestrial exo-planets requires a coronagraph. Several coronagraph architectures have been poposed ${ }^{1-4}$. Typical designs employ low F\# large aperture telescopes necessary to collect and focus radiation. Direct imaging of terrestrial exo-planets requires a telescope/instrument system capable of controlling and suppressing unwanted star light to less than one part in $10^{-}$ ${ }^{10}$ over the wide wavelength band pass spanning the near UV to near IR. Radiation incident on the large primary mirror becomes partially polarized upon reflection.

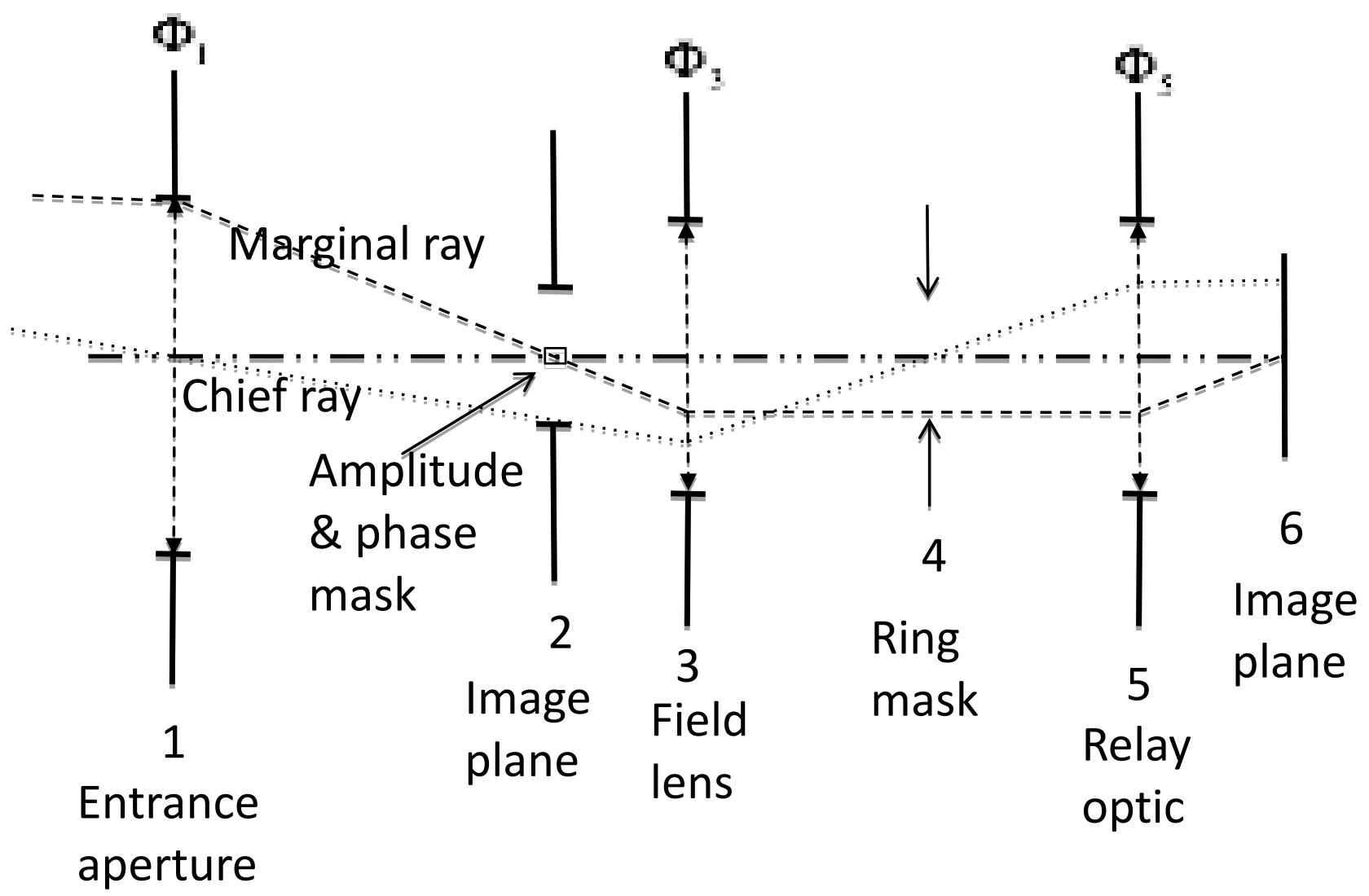

Figure 1. Schematic view of an imaging coronagraph-telescope. The coronagraph is used to control scattered light in optical systems built for high contrast stellar astronomy applications such as exoplanet and binary star atmospheric interaction research. 
Breckinridge and Oppenheimer ${ }^{5}$ showed that polarization introduced in the telescope-coronagraph system increases scattered light to limit system performance. There are two sources of polarization: One is characteristic to the natural curved shape of the primary mirror, we call the Fresnel polarization ${ }^{7}$; the other is characteristic of the microstructure of high reflectivity coatings required for high efficiency and this we call the anisotropy polarization. The curvature of the mirror introduces a polarization apodization across the pupil ${ }^{5,7}$, to produce aberrations, which, in turn affects the system image quality and lowers contrast. Spatially non-uniform anisotropies in the deposited thin film structure introduce unwanted phase and amplitude errors on the wavefront to increase scattered light, degrade image quality and lower contrast. This paper addresses an innovative mitigation technique that will significantly reduce the effects of both Fresnel polarization and unwanted polarization effects introduced by spatially non-uniform thin film anisotropies.

Fresnel polarization is introduced when a ray reflects from a tilted surface ${ }^{7}$. The reflectivity $R_{p}$ for that portion of the incident ray whose electric vector is parallel to the plane of incidence is less than that $\left(R_{s}\right)$ for the ray whose vector is perpendicular to the plane of incidence. For a concave telescope mirror, the marginal ray angle deviation $\theta$ is given by

$$
\theta=\arctan \left\{\frac{1}{2 F \#}\right\}
$$

For a typical space telescope with $\mathrm{F} \#=1.3$, the angle is then about 20 degrees. At this angle, for the case of the ray reflecting from a silver substrate, $\sim 2 \%$ of the light is polarized. This means that at the highest angular resolution of the system $\sim 2 \%$ of the light at the edge of the pupil is not contributing to an image, but rather to the background.

\subsection{Polarization Analysis}

There are several methods used to characterize the polarization in an optical system and optical devices. All of these methods are useful, (or not so useful) depending on specific quantatative applications. Jones calculus ${ }^{8-9}$, which characterizes a birefringent network, is a powerful technique in which the state of polarization is represented by a twocomponent column vector, and each optical element (or layer) is represented by a $2 \mathrm{x} 2$ matrix. The stokes vector and Muller matrix are based on the same physical approximations as the Jones vector ${ }^{8-9}$, but relates to the irradiance the detector sees and is often used in remote sensing applications. The Stokes vector and Muller matrix also include depolarization explicity in the matrix. Since both Jones and Muller calculus are based on the same physics models, both methods are limited to normally incident and paraxial rays only. For example, the two polarization states of light incident on an anisotropic material (mirror, coating, and SVRP layer) are in general not mutually orthogonal for off axis light. Both the Jones and Muller calculus methods neglect the Fresnel refraction and reflection at the surfaces. These methods do not offer an explanation of the light leakage for off axis light. Hence other polarization techniques such as the extended Jones vector, Berreman's 4x4 matices, or Finite Difference Time Domain (FTDT) are used instead ${ }^{10}$.

\subsection{Extended Jones and Berreman's 4x4 Method}

The telescope primary mirror in a coronoagraph system for space flight is typically a low F\#. These low F\# systems require a steeply curved mirror which introduces a spatially varying polarization effects across the aperture (or exit pupil). To accurately calculate the polarization effect, the effect of reflection at the highly reflecting metal coating interfaces cannot be ignored. The Berreman $4 \times 4$ method $^{10-12}$ is a powerful technique for treating the transmission of light in a complex system by treating the polarization as a general birefringnet network. Exact solution can be obtained by the $4 \times 4$ matrix method as discussed in the literature ${ }^{10-12}$. The $4 \times 4$ matrix method takes into account the effect of refraction and multiple reflections between interfaces. If the effect of multiple reflections can be neglected, the Berreman's method reduces to the extended Jones matrix methods ${ }^{11}$. The extended Jones matrix is easier to manipulate mathematically and yet accounts for the Fresnel refraction and single reflection at the interfaces. For this paper we use the extended Jones for the telesope mirror and the Berremans method for the SVRP device. 
The Berreman 4x4 matrix method relates to the propagation of polarized light to stratified media that are uniform in their dielectric properties in one plane, which is taken to be the xy plane. The method is discussed in this section and in more detail in the literature ${ }^{8-12}$. The components of the electric field $\mathrm{E}$ and the magnetic field $\mathrm{H}$ in the plane of the layer can be solved from Maxwell's equations as:

$$
\left[\begin{array}{c}
E_{x}(x, y, z, t) \\
\mu_{0} c H_{y}(x, y, z, t) \\
E_{y}(x, y, z, t) \\
-\mu_{0} c H_{x}(x, y, z, t)
\end{array}\right]=\boldsymbol{\psi}(z) e^{-i \omega\left(t-\frac{\eta x}{c}\right)}
$$

Where $\boldsymbol{\psi}(z)$ is a column vector and where the angular frequency $\omega$ is related to the wavelength in vacuum $\lambda$ and the speed of light c by:

$$
\omega=c k=\frac{2 \pi c}{\lambda}
$$

Here, $\mathrm{k}=2 \frac{\pi}{\lambda}$ is the magnitude of the wave vector in a vacuum. For the sake of convenience, the $\mathrm{x}$ axis is chosen such that the light wave properties in the xz plane which the plane of incidence. The $\mathrm{x}$ component of the wave vector is equal to $\frac{\eta \omega}{c}=\eta k$, meaning that $\eta$ is proportional to this in plane wave vector component. The column vector $\boldsymbol{\psi}$ satisfies the Berreman equation:

$$
\frac{d \psi}{d z}=i k D \cdot \psi
$$

where $\mathrm{D}$ is a $4 \mathrm{x} 4$ matrix generally referred to as the Berreman matrix (or simply the $4 \mathrm{x} 4$ matrix). Assuming that the magnetic susceptibility can be neglected, the optical properties of the dielectric can be described by the dielectric tensor with components $\epsilon_{\alpha \beta}(\alpha, \beta=x, y, z)$. Then the following expression for the Berreman's matrix $\mathrm{D}$ is

$$
D=\left[\begin{array}{cccc}
-\eta \frac{\epsilon_{z x}}{\varepsilon_{z z}} & 1-\frac{\eta^{2}}{\epsilon_{z z}} & -\eta \frac{\epsilon_{z y}}{\epsilon_{z z}} & 0 \\
\epsilon_{x x}-\frac{\epsilon_{x z} \epsilon_{z x}}{\epsilon_{z z}} & -\eta \frac{\epsilon_{x z}}{\epsilon_{z z}} & \epsilon_{x x}-\frac{\epsilon_{x z} \epsilon_{z x}}{\epsilon_{z z}} & 0 \\
0 & 0 & 0 & 1 \\
\epsilon_{x x}-\frac{\epsilon_{x z} \epsilon_{z x}}{\epsilon_{z z}} & -\eta \frac{\epsilon_{z x}}{\varepsilon_{z z}} \epsilon_{x x} & -\frac{\epsilon_{x z} \epsilon_{z x}}{\epsilon_{z z}} & 0
\end{array}\right]
$$

Equation 3 and 4 are the central equations of the Berreman's $4 \times 4$ matrix method. There are several ways to solve numerically as well as analytically ${ }^{11-12}$. We follow the Eidner-Olano ${ }^{13-15}$ method.

\section{Fresnel Effects of the Telescope Primary Mirror}

Breckinridge and Oppenheimer ${ }^{5}$ showed that polarization introduced in the telescope-coronagraph system increases scattered light to limit system performance. There are two sources of this unwanted polarization: one is characteristic to the natural curved shape of the primary mirror, which we call the Fresnel polarization. The other is an anisotropic characteristic of the deposition process of the high reflectivity metal coatings required for high efficiency. This anisotropy results in unwanted birefringence, which we call the anisotropy polarization. Spatially non-uniform anisotropies in the deposited thin film introduce unwanted phase and amplitude errors on the wavefront to increase scattered light, degrade image quality and lower contrast. The curvature of the mirror introduces a polarization apodization across the pupil ${ }^{2,3}$ resulting in aberrations, which, in turn also effects the system image quality and lowers contrast.

The Berreman's method can be extended to compute the polarization apodization in terms of contrast and retardance, as a function of angle of incidence $\theta$ and wavelength $\lambda$, across the aperture (or corresponding pupil). The metallic mirror is 
approximated as a perfect conductor. In such a perfect conductor any electric field coming from external sources will be cancelled by the rearrangement of the freely moving charges. As a consequence the net electric field in the mirror must be zero, and at the interface $\mathrm{E}_{\mathrm{x}}=\mathrm{E}_{\mathrm{y}}=0$.

For real metals the finite conductivity $\sigma$ must be taken into account. As a consequence the dielectric constant $\epsilon$ must be replaced by $\epsilon+\frac{i \sigma}{\epsilon_{0} \omega}$, thus, the refractive index $n=\sqrt{\epsilon+\frac{i \sigma}{\epsilon_{0} \omega}}$ is complex ${ }^{11}$. For sufficiently large $\sigma$ the electric field is inversely proportional to the square root of $\sigma$, which implies that in the limit of a perfectly conducting metal $(\sigma \rightarrow \infty)$ the electric field at the interface is restricted to the case of an ideal metallic mirror. Using this Berreman's extension, we define $S_{1}$ as,

$$
S_{1}=\frac{H}{V}
$$

where $\mathrm{H}$ is the intensity measured for the light polarized horizontally and $\mathrm{V}$ is the intensity measured for the vertically polarized light. Figure 2(a) shows a three dimensional view of a plot of Log to the base 10 of $S_{1}$ as a function of the light beam incidence angle, from zero to thirty degrees onto a tilted thin film aluminum coated mirror and as a function of wavelength. The extended Berreman's $4 \mathrm{x} 4$ matrix approach can be used to calculate the retardance as a function of angle of incidence $\theta$ and wavelength $\lambda$ as shown in Figure 2(b). Referring to figure 2(b), the phase angle in degrees on the vertical axis between the $\mathrm{H}$ and the $\mathrm{V}$ components as a function of the incidence angle, from zero to thirty degrees on a tilted thin film aluminum coated mirror and as a function of wavelength. These Figures show the significance of the birefringence and phase on reflection from a highly reflecting metal film. Our model agrees very favorably with Breckinridge and Oppenheimer's results ${ }^{5}$ as shown in Figure 2(a). Moreover, our model provides the retardance induced by the mirror as shown in Figure 2(b). Figure 4 and 5 shows the significance of the birefringence and phase on reflection from a highly reflecting metal film. Although these graphs are for an ideal mirror, we can design a compensator for any polarization state and fabricate it in the ONSL.
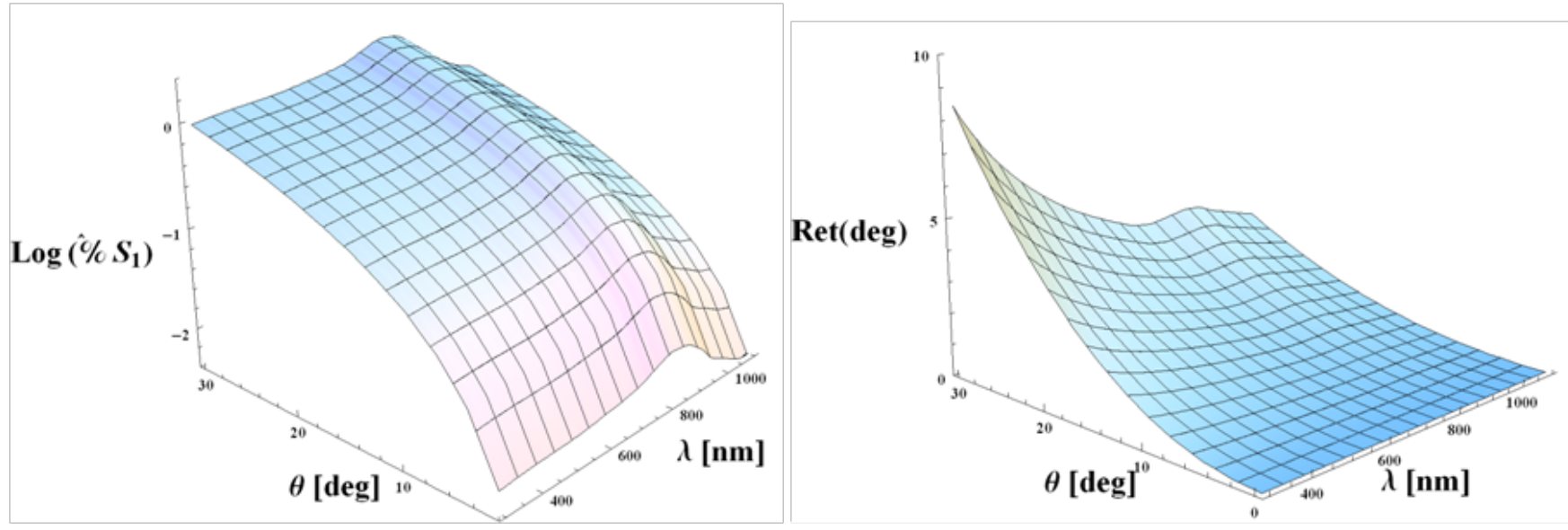

Figure 2. Polarization contrast (left) and retardance (right) as a function of angle of incidence $\theta$ and wavelength $\lambda$. The plots represent a horizontal "slice" through half of the mirror. 


\section{Mitigating Fresnel Polarization using a spatially varying retardance plate (SVRP)}

A spatially varying retarder plate (SVRP) can be placed at a convenient pupil plane, such as the ring mask plane 4 shown in figure 1. Our SVRP consists of a stack of nanolayers of anisotropic and/or isotropic materials $\mathrm{P}_{1}, \mathrm{P}_{2}, \mathrm{P}_{3} \ldots \mathrm{P}_{\mathrm{n}}$ as illustrated in figure 3. Each layer has it's slow axis oriented at an azimuth angle $\gamma_{1}, \gamma_{2}, \gamma_{3}, \ldots \gamma_{n}$

Optical birefringence describes the difference of a material's refractive index with direction. When the birefringence is on the order of the change of the in-plane refractive index between adjacent materials, surprising and useful optical effects occur.

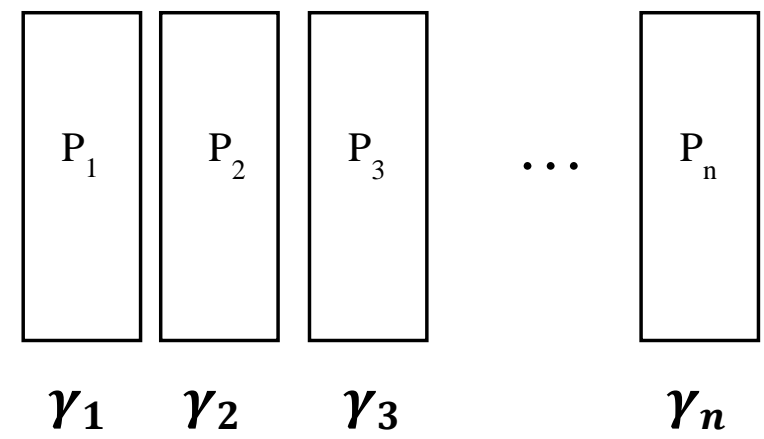

Figure 3. Synthesized Optical material (a). A stack of anisotropic and isotropic material $\mathrm{P}_{1}, \mathrm{P}_{2}, \mathrm{P}_{3} \ldots \mathrm{P}_{\mathrm{n}}$ can be stacked. Each layer thickness, birefringence, and orientation is layered to produce a custom polarization compensator. The SVRP consists of a pixelated array of these stacks as shown in (b). The resolution of each stack can be as small as 0.6 micron and with the appropriate boundary conditions the SVRP can act as a continuous spatially varying sheet or a discrete pixelated device.

We are investigating a wide variety of birefringent materials and fabrication methods for developing the SVRP device to determine the most suitable to enable $>10^{12}$ contrast. One such material is $\mathrm{MgF}_{2}$. We first consider a single layer $\mathrm{MgF}_{2}$. For multi layers, a device of Quartz is useful. Quartz has a transparency range extending from $0.25 \mu \mathrm{m}$ to $2.5 \mu \mathrm{m}$ ( $>99 \%$ ) which is suitable bandwidth of interest in coronagraphs. $\mathrm{MgF}_{2}$ is transparent over an even broader spectral range. Both Quartz and MgF2 are birefringent, but quartz has a much smaller birefringence than MgF2. Hence these two materials are often used in tandem, for example in the design of achromat waveplates. Both materials are widely used in high-performance optical devices and systems and for coatings. We are evaluating several materials in the design and fabrication process including quartz and $\mathrm{MgF}_{2}$. Quartz and MgF2 both are commonly used in high performance optical systems and are known to have high transmittances $>90 \%$ over $300 \mathrm{~nm}$ to $1200 \mathrm{~nm}$. In addition the very low scattering making them a suitable candidate for the SVRP. Other materials we discuss in section 4.0 include liquid crystals, polyimide, and photo polymers.

\subsection{Optical Nanomaterial Synthesis Design Methodology}

To design a SVRP, we first must consider the stack of dielectric (birefringent or isotropic layers) as shown in figure 3. The Berreman's method can be used to compute a propagator matrix Pj for each layer in the stack. The propagator matrix can be expressed in terms of $\mathrm{D}$ as

$$
P=\exp (i k D)=\sum_{l=0}^{\infty} \frac{(i k d)^{l}}{l !} D^{l}
$$


Where the Taylor series of the exponential function is used to define that function for the matricies. The Matrix D can be written in the diagonal form as

$$
\begin{aligned}
& D=T \bar{D} T^{-1} \\
& T_{k l}=\psi_{k}^{(l)} \\
& \overline{D_{l m}}= \begin{cases}\mu_{l} & \text { if } l=m \\
0 & \text { if } l \neq m\end{cases}
\end{aligned}
$$

Clearly D can be expressed in terms of the eigenvalues of D, whereas $\mathrm{T}$ can be expressed in terms of the eigenvectors of D. Substitution to equation (2) gives:

$$
\begin{gathered}
P=T \bar{P} T^{-1} \\
\bar{P}_{l m}=\left\{\begin{array}{c}
\exp \left(i k \mu_{l} d\right) \text { if } l=m \\
0 \quad \text { if } l \neq m
\end{array}\right.
\end{gathered}
$$

Another relatively recent $4 \mathrm{x} 4$ matrix method for general biaxial media, differing form Berreman's method has been proposed by Yuan and co-workers ${ }^{13}$. As noted in the literature, the two methods are equivalent, as both are based on the exact solutions to Maxwell's equations. In particular, the quartic equation for the $\mathrm{z}$ component of the wave vector $\mathrm{k}_{\mathrm{z}}$ given by Yuan corresponds to the quartic equation of the eigenvalues of the Berreman matrix $\mu$, because these eigenvalues are equal to $\mathrm{k}_{\mathrm{z}} / \mathrm{k}$.

For the stack of dielectric (birefringent or isotropic) layers shown in figure 3, each layer is labeled 1,2,3..,N the $4 x 4$ propagation matrix $\mathrm{P}_{\mathrm{j}}$ of each individual layer can be calculated using equation 2. In order to find the overall propagation matrix $\mathrm{P}$ of the stack of dielectric layers, boundary conditions at the interface between two dielectric layers $\mathrm{m}$ and $\mathrm{n}$ are needed. According to Maxwell theory the components of the electric and magnetic fields $\mathrm{E}$ and $\mathrm{H}$ parallel to the interface between two dielectric medial $\mathrm{m}$ and $\mathrm{n}$ must be continuous, provided that no charge or currents are present at the interface. As a consequently $E_{x}, E_{y}, H_{x}, H_{y}$ are continuous at the interface, ie the column vector $\psi$ satisfies the boundary condition

$$
\begin{aligned}
& \boldsymbol{\psi}_{+}=P \cdot \boldsymbol{\psi}_{-} \\
& \mathrm{P}=\mathrm{P}_{\mathrm{n}} \ldots \mathrm{P}_{3} \mathrm{P}_{2} \mathrm{P}_{1}
\end{aligned}
$$

Here $\boldsymbol{\psi}_{+}$and $\boldsymbol{\psi}_{-}$are the Berreman column vectors at the two sides of the stack of dielectric layers

\subsection{Design Example of a SVRP}

The design of a spatially varying retarder plate (SVRP), requires careful consideration of the material properties and fabrication techniques. Not only does the birefringence vary spatially across the mirror, but the orientation of the polarization state varies spatially as well, as depicted in figure 4(a). The orientation of the polarization as a function of position is shown in Figure 6 (a). Referring to the figure, two arbitrary small regions P1 and P2 have different birefringence and different polarization orientations. For example the alignment layer can be in correspondence with the polarization orientation of the reflected light off the mirror at a pupil plane as depicted in figure 4(b). The birefringent stack consisting of layer $B_{1}, B_{2} . . B_{n}$, as shown in figure 4 (c). The nanolayers are stacked and the stacking arrangement varies spatially (to a resolution of 0.6 microns for our fabrication process discussed in section 4) to yield the desired 
polarization compensation to mitigate the Fresnel effects. The final istortipic and amplitude layers compensate for the wavefront variation and amplitude variation repectively to yield a uniformly, optically flat, wavefront in a uniform polarization state. Although the design depicted in figure 4 illustrates the case with the alignment layer is in the direction of the polarization state of the aperture (or pupil), in general the orientation of the alignment layer correspond to the design of the birefringent stack used to compensate for the Fresnel polarization.

Consider the marginal ray of an F\#=1 Telescope that has a 30 degree angle of incidence. This represents the worst case scenario of a telescope system. 2D plot of the retardance vs wavelength from figure 2(b) is shown if figure 5(a). Referring to the figure, the telescope has approximately 9 degrees of maximum retardance (around $300 \mathrm{~nm}$ in the figure) for a 30-degree angle of incidence. The retardance verses wavelength for a 15 degree angle of incidence is shown in Figure 5(b), which has 2 degrees of retardance.
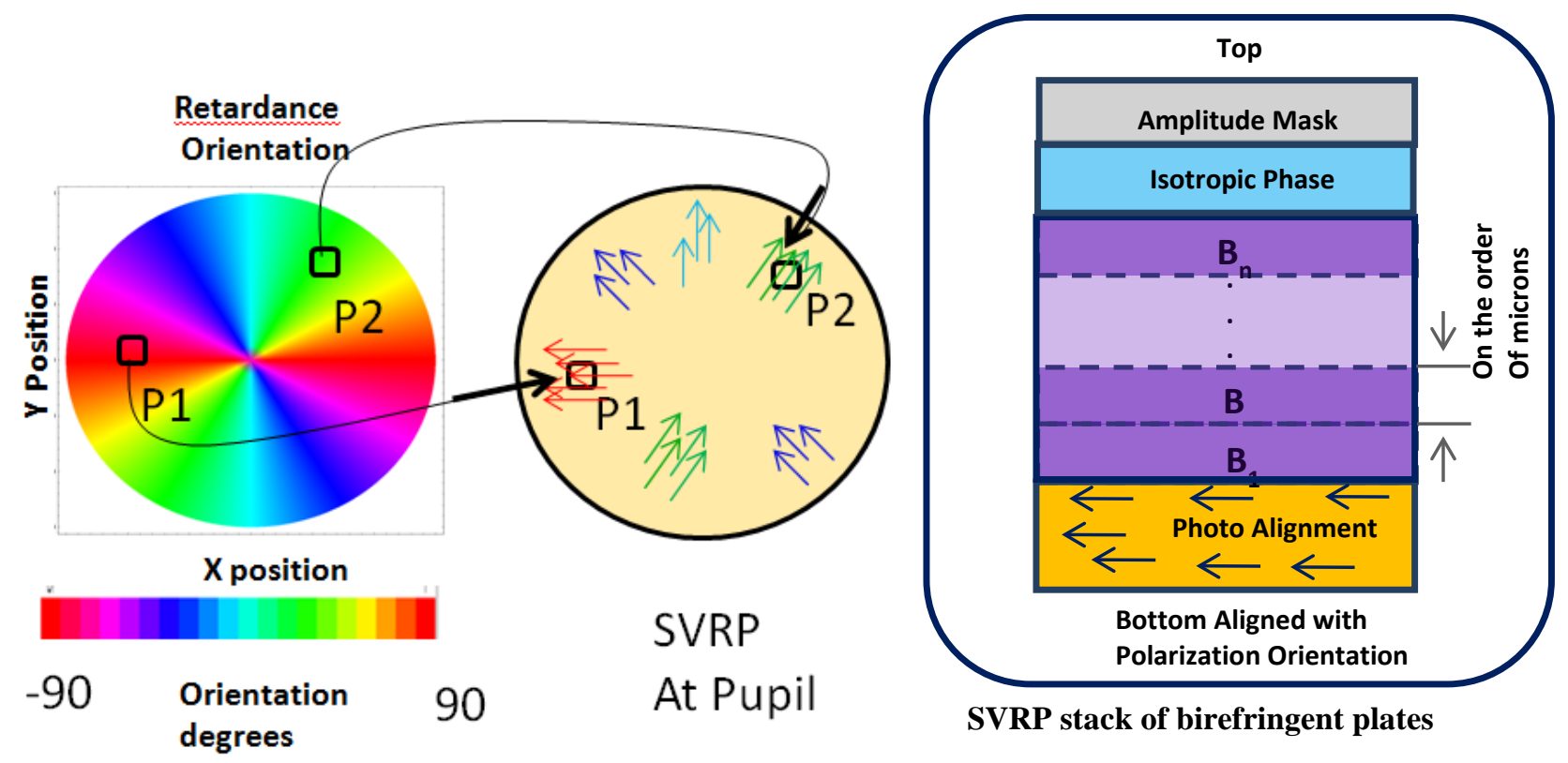

Figure 4. Photo alignment layer of a SVRP plate. (a) shows a spatially variable retarder plate (SVRP) face on (x,y) with retardance direction indicated by the colors shown in the stripe below which maps color into orientation in degrees as shown. (b) shows two particular regions, P1 and P2 which have two different polarization states and orientation are shown. (c) shows a diagram of the typical stack or sandwich. The bottom layer is a layer of homogeneous dielectric oriented to the polarization direction. Birefringent layers of $B_{1}, B_{2} \ldots B_{n}$ will be deposited with thickness layers and specific process recipe calculated and optimized to compensate for the Fresnel polarization of light reflected form the telescope.

For The Contrast $\left(I_{\max }-I_{\min }\right) /\left(I_{\max }+I_{\min }\right)$ of a coronagraph system is related to the diattenuation and retardance by

$$
\text { contrast }=K(1-\cos (\Gamma))
$$


For a simple 1 layer SVRP plate the polarization compensation for the 30 degree and 15 degree angle of incidence is shown in figure 6. Since the dispersion of a nano layer is very small, the polarization compensation is not that dependent on the material selection. Hence, other material will result in comparable polarization compensation as that shown in figure 6. The material selection is however very important for consideration of clarity, transmission, alignment, fabrication, thermal and space-radiation effects.
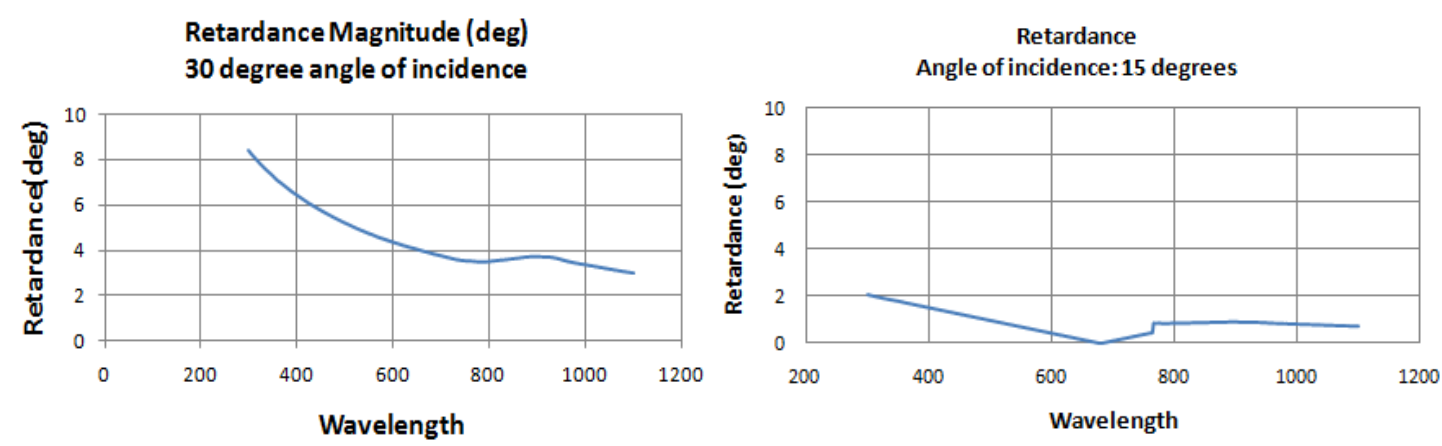

Figure 5. Retardance as a function of wavelength (a) at an angle of incidence 30 degrees (b) at and angle of incidence of 15 degree
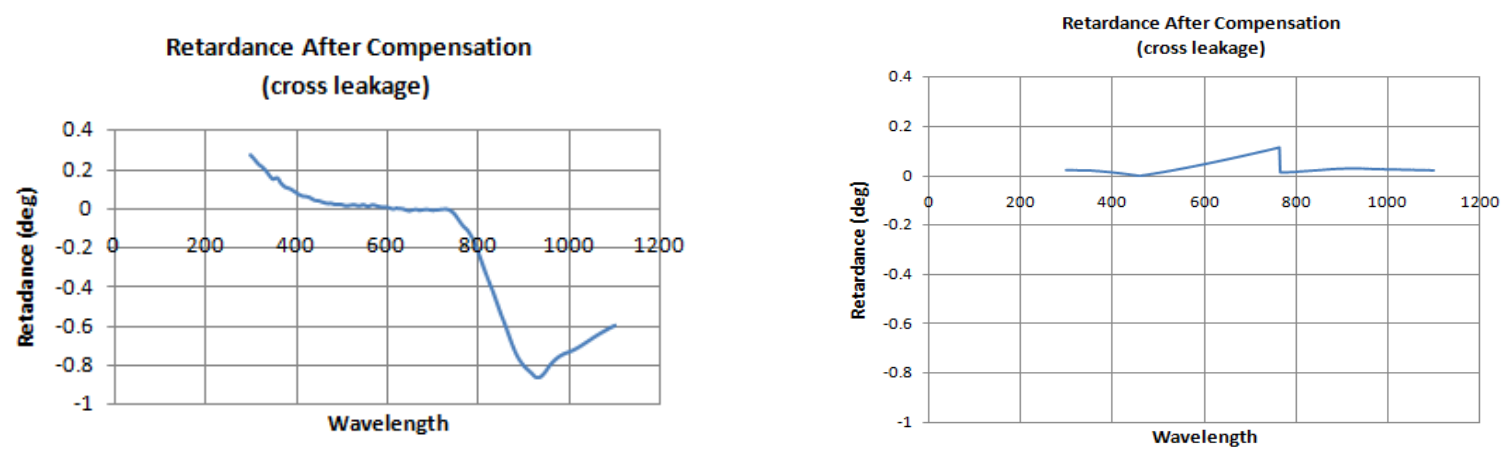

Figure 6. Single cell design of MgF2. (a) For an angle of incidence of 30 degrees retardance as a function of wavelength and after compensation by a single layered device reduces the retardance to better than 0.01 degree over $500-700 \mathrm{~nm}$. Note the scale is $1 / 10$ that of Figure 6.

\section{Fabrication and Experimental Results.}

The ability of the birefringent layers to orient in a particular direction when in contact with a specially prepared surface is a phenomenon of major importance other SVRP plate fabrication. There are several methods we use in our NASA OSNL laboratory. Our lab has made significant progress in the development of photo aligned materials. Manufacturers require alignment layers with a wide range of optimized properties. Thin films must easily be formed with high 
photosensitivity in order to facilitate rapid processing and also to avoid unwanted degradation. The resultant photoaligned film must be insoluble and thermally, electrochemically and photo-chemically stable.

\subsection{Photo-alignment using photo degradation}

The successful photo-alignment of a polyimide is considered to be an important technological goal, because of the high thermal stability of polyimides and their acceptance as the alignment layer of choice by the LC display industry. Hasegawa and Taira ${ }^{\mathrm{x}}$ first reported photo-alignment of polyimide by polarized light exposure at $257 \mathrm{~nm}$. Homogeneous LC alignment was obtained in a direction perpendicular to the polarization of the incident UV beam. This is the direction of the maximum density of unbroken polyimide chains on exposure. Therefore, alignment was attributed to the anisotropic depolymerization of the polyimide. Further studies confirmed this and the alignment direction was shown to switch by varying the polarization direction of the aligning beam ${ }^{18}$.

We used Nissan SE-7492 in the form of polyamic acid. The polyimide film was spin-coated onto a quartz substrate. It was cured at $2500 \mathrm{C}$ for one hour or 200 degrees C for an hour and a half. A $200 \mathrm{~W} \mathrm{Hg}$ (Xe) lamp equipped with a dichroic mirror reflecting form 230 to $430 \mathrm{~nm}$, was used as the light source. A dichroic UV linear polarizer was used to obtain the linearly polarized UV light. The average power density for the un polarized and polarized light was approximately 40 and $10 \mathrm{~mW} / \mathrm{cm}^{2}$, respectively, measured using a radiant energy meter.

The transmittance curve from this rudimentary spectroscopic ellipsometer can be processed along with a simple model to determine the dispersion of the birefringence. The standard expression for an $a$-plate with positive birefringence between crossed polarizers applies $^{8-9}$ :

$$
T=\frac{1}{2} T_{0} \sin ^{2}(2 \theta) \sin ^{2}\left(\frac{\pi \Delta n d}{\lambda}\right)
$$

where $T_{0}$ is the unpolarized irradiance incident on the first polarizer, $\lambda$ is the wavelength, $d$ is the film thickness, $\Delta n$ is the birefringence, and $\theta$ is the angle between the effective optical axis of the birefringent film and the transmission axis of either polarizer always set to $45^{\circ}$ in our geometry) Since $T$ is a periodic function involving a square root, the value of $\Delta n$ cannot be unambiguously determined without some additional steps. We have used two methods to find the dispersion of the birefringence from the transmittance. The first method involves a direct computation from the data as follows. From eq (7), the birefringence can be expressed as:

$$
\Delta n=\frac{\lambda}{\pi d}\left[m \pi \pm \sin ^{-1}\left(\sqrt{\left.2 T / T_{0}\right)}\right]\right.
$$

Where $\mathrm{m}$ is a non negative integer that describes the order of the solution. From basic principles of these optically transparent materials, we know that $\Delta n$ is continuous across the visible spectrum. Hence, the sign of the $\sin ^{-1}$ function and the order $\mathrm{m}$ are constant between each maximum and minimum in $\mathrm{T}$. Then one can simply manually or computationaly choose both parameters for each data point such that the ambiguity is resolved and a continuous solution $\Delta n(\lambda)$ is found.

We often use a second method, that is very useful in optical system design ${ }^{8-9}$, that involves an indirect solution that requires the least-squares-fit of a dispersion model to the transmittance. Since our polymer films are optically transparent throughout the visible region, the refractive index can be modeled by the first-order Sellmeier dispersion ${ }^{17}$ relation:

$n(\lambda)^{2}-1=a+b \lambda^{2} /\left(\lambda^{2}-\lambda_{0}^{2}\right)$, where $a, b$, and $\lambda_{0}$ are constants. Granting the standard assumptions, this can be reduced to the slightly simpler Cauchy formula: $n(\lambda)=A+B / \lambda^{2}$, where $A$ and $B$ are constants for a given material.

The dispersion of our birefringent films is therefore modeled by the following equation:

$$
\Delta n(\lambda)=n_{e}(\lambda)-n_{o}(\lambda) \approx \Delta n_{\infty}+\frac{c}{\lambda^{2}}
$$

where $\Delta n_{\infty}$ is the birefringence at long wavelengths and $C$ is a constant. Note that the absolute values of the ordinary (no) and extraordinary ( $\left.n_{e}\right)$ indices are not required to determine $\Delta n(\lambda)$. In order to calculate the dispersion of the 
birefringence, we combine Eq. (7) and Eq. (9) and utilize least squares minimization to find the parameters $\Delta n_{\infty}$ and $C$ that give the closest fit to our transmittance data.

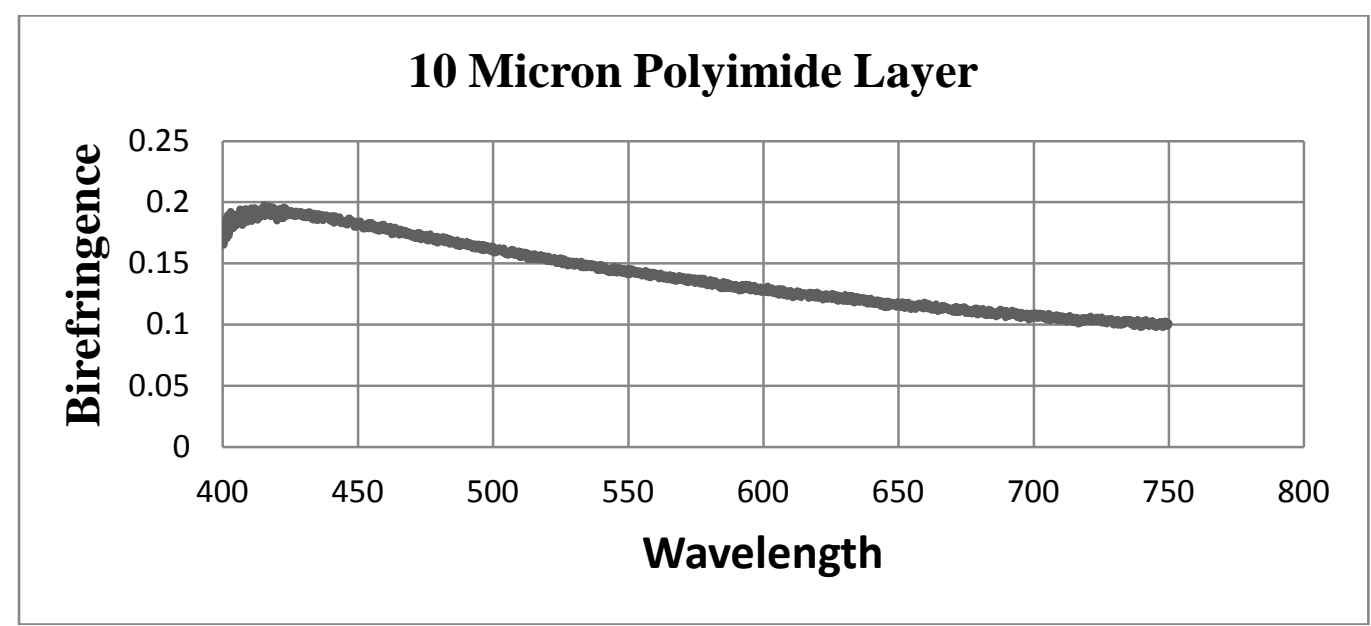

(a)

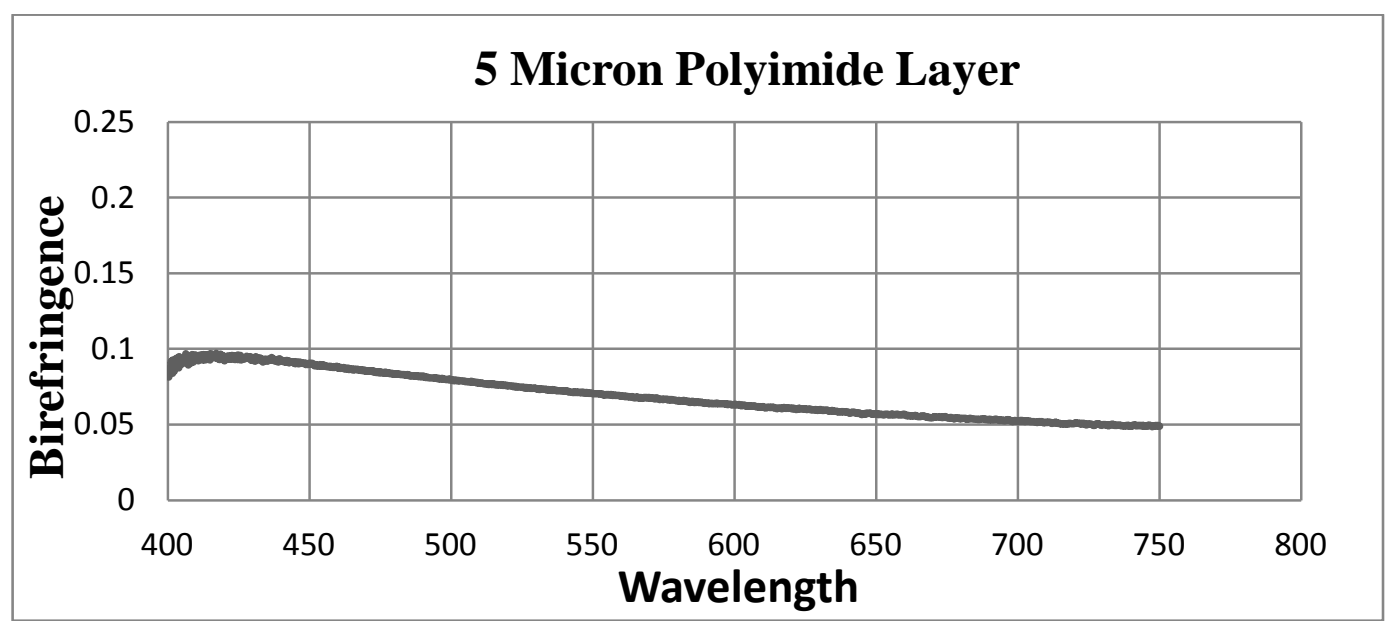

(b)

Figure 7. Representative data for uniaxial polyimide film: (a) transmittance between crossed polarizers and (b) birefringence calculated using equation (8).

\subsection{PhotoAlignment using Polymerized Photo Polymer.}

Another interesting method we are currently investigating is an aligning method where photopolymers are polymerized with linearly polarized light ${ }^{19}$. The main advantage of this method is that it allows different orientations on the same glass or silicon substrate. Several polymers were tried and two such polymers are: poly(vinyl 4-methoxy cinnamate $^{18}$ and poly(vinyl cinnamate) ${ }^{19}$ showed some birefringence.. Both polymers exhibit a depletion cinnamic acid side chain molecules along the direction of polarization, increasing in the mean time the number of photoinduced cyclo additions along a direction perpendicular to the polarization direction. Therefore, pre-irradiated isotropicallyoriented molecules change into an anisotropic distribution of photopolymerized side chains, which can induce a de ${ }^{-}$nite orientation of liquid crystal molecules by means of their in-line aromatic rings. These methods are very low technical readiness level and hence require more research and development, which we are pursuing at our LaRC nano optic facilities for use in exoplanet missions and will be reported in future publications. 


\section{REFERENCES}

[1] Ford, V. et al., TPF-C Flight Baseline Interim Report, http://planetquest.jpl.nasa.gov/documents/TPFCFB1_Report.pdf

[2] Belikov, R. et al., Demonstration of High Contrast in 10\% Broadband Light with the Shaped Pupil Coronagraph, Proc. SPIE Vol 6693-36 (2007).

[3] Kasdin, N.J., R. J. Vanderbei, D. N. Spergel, and M. G. Littman, "Extrasolar planet finding via optimal apodizedpupil and shaped-pupil coronagraphs,” The Astrophysical Journal 582, pp. 1147-1161, January (2003).

[4] Guyon, O., et al., "Exoplanet Imaging with a Phase-induced Amplitude Apodization Coronagraph. I. Principle", ApJ, 622, 744-758 (2005).

[5] Breckinridge, J. B. and B. R. Oppenheimer "Polarization effects in reflecting coronagraphs for white-light applications in astronomy", Astrophys. J. 600: 1091-1098, (2004).

[6] Clark, N "Design and performance evaluation of sensors and actuators for advanced optical systems" SPIE March 2011.

[7] Born, M., and E. Wolf, Principles of Optics, Chapter 14, (2005).

[8] Brosseua, C "Fundamentals of Polarized Light: A Statistical Optics Approach”, John Wiley \& Sons, 1998.

[9] Goldstein, D., “Polarized Light. Third Edition”, CRC Press 2010.

[10] Hodkinson,I and Wu, Q “ Birefringent Thin Films and Polarizing Elements” World Scientific Press, 1998.

[11] Berraman D. "Optics in Stratified and Anisotropic Media: 4x4 Matrix Formulation, J Opt Soc. Am 502, pp502-510 April (1972).

[12] D. W. Berreman: "Optics in smoothly varying planar structures: Application to liquid-crystal twist cells“, J. Opt. Soc. Am., 63, 1374-1380 (1973)

[13] Eidner, K, Mayer G., Schmidt M., and Schmiedel H., "Light propagation in stratified anisotropic media: orthoganlity and symmetry properties of the 4x4 matrix formalisms” Mol Cryst. Liq Cryst. 172, 191 (1989).

[14] Eidner K. "Light propagation in stratified anisotropic media: orthoganlity and symmetry properties of the $4 \times 4$ matrix formalisms”,J. Opt. soc. Am. A 616571989

[15] Oldano C. "Electromagnetic wave propagation in Anisotropic stratified media” Phys. Rev A 40 6014-6020 (1989).

[16] Eidner, $\mathrm{K}$ e.a.: "Optics in stratified media - the use of optical eigenmodes of uniaxial crystals in the $4 \times 4$-matrix formalism”, Mol. Cryst. Liq. Cryst., 172, 191-200 (1989)

[17] Hasegawa M and Taira Y 1995 Proc. 21st JLCC p 344

[18] H. Y. Joo, H. J. Kim, S. J. Kim, and S. Y. Kim, Thin Solid Films 368, 67

2000.

[19]Chigrinov V, Kozenkov V, and Kwik H, “Photoalignment of Liquid Crystaline Materials”, Wiley 2008. 
Proc. of SPIE Vol. $8146814600-13$

Downloaded From: http://proceedings.spiedigitallibrary.org/ on 10/28/2016 Terms of Use: http://spiedigitallibrary.org/ss/termsofuse.aspx 